\title{
Dunford-Pettis-like Properties of Projective and Natural Tensor Product Spaces
}

\author{
Jesús M. F. Castillo and Juan A. López Molina
}

\begin{abstract}
Several properties of weakly $p$-summable sequences and of the scale of $p$-converging operators (i.e., operators transforming weakly $p$-summable sequences into convergent sequences) in projective and natural tensor products with an $l_{p}$ space are considered. The last section studies the Dunford-Pettis property of order $p$ (i.e., every weakly compact operator is $p$-convergent) in those spaces.
\end{abstract}

\section{INTRODUCTION}

In this paper several properties of the scale of p-converging operators in projective and natural tensor products with an $l_{p}$ space are considered. This scale, introduced in [2] and [3], is intermediate between the ideals of unconditionally converging operators and the ideal of completely continuous or Dunford-Pettis operators. Since p-converging operators are characterized by the property of sending weakly-p-summable sequences into convergent ones, a part of the study is devoted to a special class of subsets of vector sequence spaces, termed almost compact sets, nontrivial examples of which are, in certain spaces, precisely the weakly-p-summable sequences, $1 \leq p<+\infty$. Section 2 characterizes the compact sets of $l_{p} \hat{\otimes}_{\pi} X$ and $l_{p} \hat{\otimes}_{\Delta_{p}} \mathrm{X}$, extending results of Leonard [8] and Bombal [1]. Section 3 considers the Dunford-Pettis properties of order $p$ in projective and natural tensor product spaces of $l_{p}$ and a Banach space $X$. For $l_{p}$-sums of sequences of Banach spaces, generalizations of results of Bombal [1] are obtained. Those properties were introduced in [3].

1991 Mathematics Subject Classification: 46B20, 46B28, 46B45.

Editorial Complutense. Madrid, 1993. 


\section{BACKGROUND}

Throughout the paper $p^{*}$ denotes the conjugate number of $p$. We base our approach to the properties of natural and projective tensor products on the use of the representations of those spaces as sequence spaces. A sequence $\left(x_{n}\right)$ in a Banach space $X$ is said to be weakly-p-summable $(p \geq 1)$ if for every $x^{*} \in X^{*}$ the sequence $\left(x^{*}\left(x_{n}\right)\right)$ is in $l_{p}$; equivalently (see [7] 19.4), if there is a constant $C>0$ such that, for each $\left(\xi_{n}\right)$ in $l_{p^{*}}$, $w_{p}\left(\left(x_{n}\right)\right)=\sup \left\{\left\|\Sigma_{n=1}^{k} \xi_{n} x_{n}\right\|:\left\|\left(\xi_{n}\right)\right\|_{p^{*}} \leq 1\right\} \leq C<+\infty$. (Here, if $p=1, c_{0}$ plays the role of $l_{\infty}$.) It is said to be absolutely-p-summable, when $p \geq 1$, if $s_{p}\left(\left(x_{n}\right)\right)=\left(\Sigma_{n \neq 1}^{+\infty}\left\|x_{n}\right\|_{1}^{p}\right)^{\frac{1}{p}}<+\infty$. (If $p=+\infty$, the $l_{p}$ norm has to be replaced by the sup norm.) It is said to be strongly-p-summable for $p \geq 1$ if $\sigma_{p}\left(\left(x_{n}\right)\right)=\sup \left\{\left|\Sigma_{n=1}^{+\infty} f_{n}\left(x_{n}\right)\right|: w_{p^{*}}\left(\left\{f_{n}\right\}\right) \leq 1,\left(f_{n}\right) \in X^{*}\right\}<+\infty$. Following [7], we shall denote by $l_{p}[X], l_{p}\{X\}$ and $l_{p}\langle X\rangle$ respectively the spaces of weakly-p-summable, absolutely-p-summable and strongly-p-summable sequences of $X$, endowed with their natural topologies: those induced by the norms $w_{p}, s_{p}$ and $\sigma_{p}$, respectively. The following isometries are wellknown (see [7] 19.4.3): $l_{p}[X]=L\left(l_{p^{*}}, X\right)$, for $1<p<+\infty$, and $l_{1}[X]=L\left(c_{0}, X\right)$. The symbols $\pi$ and $\varepsilon$ shall denote the projective and injective norms on the space $l_{p} \otimes X$ : they are, respectively, the strongest and coarsest crossnorms (i.e. norms satisfying $\|x \otimes y\|=\|x\|\|y\|$ ) which is possible to define on that space. The symbol $\Delta_{p}$ denotes the norm induced by $s_{p}$ over $l_{p} \otimes X$; the topology induced by $s_{p}$ is termed the natural topo-

logy. We shall denote by $l_{p} \hat{\otimes}_{\varepsilon} X, l_{p} \hat{\otimes}_{\pi} X$ and $l_{p} \hat{\otimes}_{\Delta_{p}} X=l_{p}\{X\}$ the comple-

tion of $l_{p} \otimes X$ with respect to $\varepsilon, \pi$, and $\Delta_{p}$, respectively. The space $l_{p} \hat{\otimes}_{\pi} X$ also admits a representation as a vector sequence space: it is the closed subspace of the space $l_{p}\langle X\rangle$ formed by those sequences which are the limit of their finite sections; this can be deduced without difficulty from [5], where it is proved that the norm $\sigma_{p}$ induces $\pi$ over $l_{p} \otimes X$.

Let $E$ be any of the spaces $l_{p} \hat{\otimes}_{\pi} X$ or $l_{p} \hat{\otimes}_{\Delta_{p}} X, p_{k}$ be the continuous projection onto the $k^{\text {th }}$ coordinate, and $i_{k}$ the canonical inclusion of $X$ into the $k^{\text {th }}$ coordinate. If $T: E \rightarrow Y$ is a continuous operator, then a sequence of operators $T_{k} \in L(X, Y)$ exists such that $T=\Sigma_{k} T_{k} p_{k}$ : explicitely, $T_{k}=T i_{k}$. We shall say that $\left(T_{k}\right)$ is the representing sequence of $T$. If $\left(X_{n}\right)$ is a sequence of Banach spaces, and $T$ is an operator from the Banach space $\left(\Sigma_{n} \oplus X_{n}\right)_{p}=\left\{x=\left(x_{n}\right) \in \Pi_{n} X_{n}:\|x\|_{p}=\left(\Sigma_{n}\left\|x_{n}\right\|^{p}\right)^{1 / p}<+\infty\right\}$ into $Y$, then the sequence $\left(T_{k}\right)$ defined by $T_{k}=T i_{k}$ is again called the representing sequence of $T$ (cf. [1]). 
We shall consider the following operator ideals: The ideal $L$ of all continuous operators; the ideal $W$ of weakly compact operators; the ideal $U$ of unconditionally converging operators, i.e., those sending weakly-1-summable sequences into unconditionally summable sequences; the ideal $K$ of compact operators; and the ideal DP of completely continuous or DunfordPettis operators, i.e., those sending weakly convergent sequences into convergent ones.

Definition. We say that an operator $T \in L(X, Y)$ is p-converging, $1 \leq p<+\infty$, if it transforms weakly-p-summable sequences of $X$ into norm null sequences of $Y$. We shall use $C_{p}$ to denote the ideal of p-converging operators.

The classes $C_{p}$ form injective, non-surjective closed operator ideals. It is not difficult to see that $C_{1}=U$ and, with the convention that the weakly$\infty$-summable are the weakly null sequences, that $C_{\infty}=D P$. A characterization of $p$-converging operators is contained in the following proposition (see [3]):

Proposition 0. Let $X$ be a Banach space, and $1 \leq p<+\infty$. If $p>1$ the operator $I d(X)$ belongs to $C_{p}$ if and only if all operators from $l_{p^{*}}$ into $X$ are compact. If $p=1, I d(X)$ belongs to $C_{1}$ if and only if all operators from $c_{o}$ into $X$ are compact.

\section{COMPACT SETS}

We shall study in Section 3 the relation between the membership of an operator $T$ in a class $C_{p}$, and the membership of the operators forming its representing sequence in that same class. To this end, we shall introduce a class of subsets which have something of the flavour of compact sets.

Lemma 1. Let $1 \leq p<+\infty$. Let $X$ and $Y$ be Banach spaces. Consider a set $A \subset l_{p} \hat{\otimes}_{\pi} X$ (resp. $A \subset l_{p} \hat{\otimes}_{\Delta_{p}} X$ ). The following are equivalent:

1. For each continuous operator $T \in L\left(l_{p} \hat{\otimes}_{*} X, Y\right)$ (resp. $\left.T \in L\left(l_{p} \hat{\otimes}_{A_{p}} X, Y\right)\right)$, the representing sequence of $T$ converges to $T$ uniformly over $A$. 
2. $\lim _{N \rightarrow+\infty} \sup _{x \in A} \pi\left[\left(x_{k}\right)_{k \geq N}\right]=0, \quad$ (resp., $\left.\lim _{N \rightarrow+\infty} \sup _{x \in A} \Delta_{p}\left[\left(x_{k}\right)_{k \geq N}\right]=0\right)$.

Proof. That $1 \Rightarrow 2$ is obvious. Let us show that $2 \Rightarrow 1$ for the case of the projective tensor product. Let $\left(x_{n}\right)$ be any sequence in $A$. Then

$$
\begin{gathered}
\left\|T\left(\left(x_{n}\right)\right)-\left(T_{1}\left(x_{1}\right), T_{2}\left(x_{2}\right), \ldots, T_{N}\left(x_{N}\right), 0,0, \ldots\right)\right\|_{Y}= \\
=\left\|T\left(0,0, \ldots, 0, x_{N+1}, x_{N+2}, \ldots\right)\right\|_{Y} \leq\|T\| \pi\left[\left(0,0, \ldots, 0, x_{N+1}, x_{N+2}, \ldots\right)\right]
\end{gathered}
$$

and this converges uniformly on $A$ by Condition 2 .

The computations for the natural product are very similar.

Definition. Let $p<+\infty$. A set $A \subseteq l_{p} \hat{\otimes}_{\pi} X$, (resp. $A \subset l_{p} \hat{\otimes}_{\Delta_{p}} X$ ), is said to be almost-compact if it satisfies either of the equivalent conditions of Lemmal.

Proposition 2. Let $p<+\infty$. A subset $A \subseteq l_{p} \hat{\otimes}_{\pi} X$, (resp. $\left.A \subset l_{p} \widehat{\otimes}_{\Delta_{p}} X\right)$, is relatively compact if and only if it is bounded, almost-compact, and its continuous projections $p_{k}(A)$ are relatively compact in $X$ for all $k \in \mathbb{N}$.

Proof. It is easy to see that all the conditions are necessary. They are also sufficient: Let $\left(x^{n}\right)$ be a sequence contained in $A \cong l_{p} \hat{\otimes}_{n} X$. Condition 1 of Lemma 1 and a diagonal argument show that a certain sub-sequence, again denoted $\left(x^{n}\right)$, exists having pointwise convergence to an element $x$. To verify that the convergence occurs in the projective norm, it is only necessary to take, in the following expression, the supremum over all elements $x^{*}$ in the unit ball of $l_{p^{*}}\left[X^{*}\right]$ :

$$
\begin{gathered}
\sum_{k=1}^{+\infty}\left|<x_{k}^{n}-x_{k}, x_{k}^{*}>\right| \leq \sum_{k=1}^{k=N}\left|<x_{k}^{n}-x_{k}, x_{k}^{*}>\right|+ \\
+\sum_{k=N+1}^{+\infty}\left|<x_{k}^{n}, x_{k}^{*}>\right|+\sum_{k=N+1}^{+\infty}\left|<x_{k}, x_{k}^{*}>\right|,
\end{gathered}
$$

and observe that the first summand can be made, for large $N$, less than $\varepsilon$; since $A$ is almost compact, the second and third summands tend to zero when $N$ tends to infinity. 
The proof for the natural product is analogous.

Remark. Lemma 1 and Proposition 2 have been proved in [1] and [8] for $l_{p}\{X\}$. The referee has informed to us that this proposition is a particular case of an old theorem due to Mazur, who stated it for the case of a Banach space having a basis, and that a more general result has been established by Goes and Welland as follows:

Theorem ([6] Thm. 1.) Let $X$ be a complete locally convex topological vector space. Let $A$ be a bounded subset of $X$ and $\left\{P_{\beta}\right\}_{\beta \in I}$ a net in $L(X, X)$. Then $A$ is relatively compact if $\left\{P_{\beta}\right\}_{\beta \in I}$ converges uniformly to the identity on $A$ and $P_{\beta}(A)$ is relatively compact for each $\beta \in I$.

Proposition 2 follows taking $P_{N}\left(\left(x_{n}\right)_{n}\right)=\left(x_{1}, x_{2}, \ldots, x_{N}, 0,0, \ldots\right)$ for $N \in \mathbb{N}$. We have left the proof of Proposition 2 for the sake of completeness.

Nontrivial examples of almost compact sets in natural and projective tensor products are provided by the next proposition.

Proposition 3. Assume that $X$ is a Banach space and that $1 \leq p, r<+\infty$. If $r<p^{*}$, then a weakly-r-summable sequence of $l_{p} \hat{\otimes}_{\pi} X$ or $l_{p} \hat{\otimes}_{\Lambda_{p}} X$ is an almost compact set. For $p=1$, a weakly null sequence of $l_{1}\{\mathrm{X}\}$ is an almost compact set.

Proof. We first show the proof for the projective product. Let $\left(a^{n}\right)$ be a weakly-r-summable sequence in $l_{p} \hat{\otimes}_{\pi} X$. Assume that $A=\left\{a^{n}: n \in \mathbb{N}\right\}$ is not an almost compact set. In that case, an $\varepsilon>0$ and two sequences $\left(n_{i}\right)$ and $\left(N_{i}\right)$ of naturals exist such that if $I_{i}$ denotes the set $\left\{N_{i}+1, \ldots, N_{i+1}\right\}$ and $P_{i}: l_{p} \hat{\otimes}_{\pi} X \rightarrow l_{p} \hat{\otimes}_{\pi} X$ denotes the projection over the indices of $I_{i}$ then

$$
\pi_{p}\left(P_{i}\left(a^{n_{i}}\right)\right)>\varepsilon .
$$

Elements $z_{i} \in\left(l \hat{\otimes}_{\pi} X\right)^{*}=L\left(l_{p}, X^{*}\right)$ with $\left\|z_{i}\right\| \leq 1$ can be chosen such that $\left|\left\langle P_{i}\left(a^{n_{i}}\right), z_{i}\right\rangle\right|>\varepsilon$. The proof of $[4, \mathrm{Thm} .1]$ shows that if $Q_{i}: l_{p} \rightarrow l_{p}$ denotes the projection over the indices of $I_{i}$, then $\left|\left\langle P_{i}\left(a^{n_{i}}\right), z_{i} Q_{i}\right\rangle\right|>\varepsilon$. Once more, the proof of $\left[4\right.$, Thm. 1] shows that the operator $B: l_{p} \hat{\otimes}_{n} X \rightarrow l_{p}$ 
defined by $B(Y)=\left(\left\langle P_{i} y, z_{i} Q_{i}\right\rangle\right)$ is continuous. By [3, Prop. 1.6.], it transforms $\left(a^{n}\right)$ into a norm-null sequence of $l_{p}$, which is a contradiction.

The proof for the natural product is essentially the same. We shall give it for the sake of completeness: If $A=\left\{a^{n}: n \in \mathbb{N}\right\}$ is not almost compact, then an $\varepsilon>0$ and two sequences $\left(n_{i}\right)$ and $\left(N_{i}\right)$ of naturals exist such that

$$
\sum_{k=N_{i}}^{k=N_{i+1}}\left\|a_{k}^{n_{i}}\right\|^{p}>\varepsilon .
$$

Normalized elements $x_{i}^{*}(k) \in X^{*}$ can be chosen such that: $\left\langle x_{i}^{*}(k), a_{k}^{n_{1}}\right\rangle=\left\|a_{k}^{n_{1}}\right\|$. If $y_{k}^{*}=x_{i}^{*}(k)$, for $N_{i} \leq k<N_{i+1}$, then $\left(y_{k}^{*}\right)$ is a bounded sequence of $X^{*}$ which defines an element of $L\left(l_{p}\{X\}, l_{p}\right)$. This operator transforms $\left(a^{n}\right)$ into a weakly-r-summable sequence of $l_{p}$, which must be norm-null (see [3, Prop. 1.6.]). Thus one has

$$
\lim _{N \rightarrow+\infty} \sup _{n \in \mathbb{N}}\left[\sum_{k=N}^{k=+\infty}\left|<y_{k}^{*}, a_{k}^{n}>\right|^{p}\right]^{\frac{1}{p}}=0,
$$

which is a contradiction.

The proof for the case $p=1$ follows closely that of the natural product, and it is only necessary to recall that $l_{1}$ has the Schur property, i.e.: weakly null sequences are norm null. That yields the proof for the projective tensor product since $l_{1} \widehat{\otimes}_{\pi} X=l_{1}\{X\}$. In other words: the statement holds for $p=1$ and $r=\infty$.

Remark. Let $X_{n}$ be a sequence of Banach spaces, and $1 \leq p<+\infty$. A set $A \subseteq\left(\Sigma_{n} \oplus X_{n}\right)_{p}$ is said to be almost compact if Conditions 1 or 2 of Lemma 1 , with suitable modifications, are satisfied. In this form, Propositions 2 and 3 can be translated to $l_{p}$-sums of sequences of Banach spaces.

\section{DUNFORD-PETTIS PROPERTIES}

A Banach space $X$ is said to have the Dunford-Pettis property $(D P P)$ if weakly compact operators defined on $X$ are completely continuous, that is, if for any Banach space $Y: W(X, Y) \subseteq D P(X, Y)$. Typical examples of Banach spaces having $D P P$ are $L_{\infty}$ and $L_{1}$ spaces. No reflexive Banach space can have $D P P$. Weakened versions of the Dunford-Pettis property were in- 
troduced in [3]. A Banach space $X$ is said to have the Dunford-Pettis property of order $p \geq 1$, if $W(X, Y) \subseteq C_{p}(X, Y)$ for all Banach spaces $Y$. We shall call this property $D P P_{p}$. Notice that $D P P_{\infty}=D P P$. Every Banach space has $D P P_{1}$. Other examples are (see [3] for details): $l_{p}$ has $D P P_{r}$ for all $r<p^{*} ; L_{p}[0,1]$ has $D P P_{r}$ for $r<\min \left\{p^{*}, 2\right\}$; Tsirelson's space has $D P P$, for all $r<+\infty$, but not $D P P$ since it is reflexive; if $i d(X) \in C_{p}$ then $C(K, X)$ has $D P P_{p}$

Lemma 4. Let $1 \leq p<+\infty$. Let $\left(X_{n}\right)$ be a sequence of Banach spaces. Assume that $E$ represents any of the spaces $\left(\Sigma \oplus X_{n}\right)_{p}$ or $l_{p} \hat{\otimes}_{\pi} X$, and that $T$ is a continuous operator from $E$ into a Banach space $Y$, having $\left(T_{k}\right)$ as a representing sequence. If $r<p^{*}$ (or $p=1$ and $r=\infty$ ), then $T$ is $r$ converging if and only if each $T_{k}$ is $r$-converging.

Proof. Let $\left(a^{n}\right)$ be a weakly-r-summable sequence of $E$. Since $\left(a^{n}\right)$ is an almost compact set, the convergence of $\left(T_{k}\right)$ to $T$ is uniform over the set $\left\{a^{n}\right\}$. Furthermore, $T_{k}\left(\left\{a^{n}\right\}\right)$ is relatively compact in $Y$ since $T_{k}$ is $r$ converging. The relationship

$$
T\left(\left\{a^{n}\right\}\right) \subseteq \sum_{k=1}^{k=N(\varepsilon)} T_{k}\left(\left\{a^{n}\right\}\right)+\varepsilon B_{Y}
$$

implies that $T\left(\left\{a^{n}\right\}\right)$ is relatively compact, and therefore $\left(T a^{n}\right)$ must be norm-null.

Remark. When $p^{*} \leq r<\infty$ the result is clearly false: simply consider the example $l_{p}\left\{l_{1}\right\}$ and $T=i d$.

Proposition 5. Let $A$ denote an operator ideal and $r<p^{*}$ (or $p=1$ and $r=\infty)$. With the same notation as in Lemma 4, $A\left(\left(\Sigma \oplus X_{n}\right)_{p}, Y\right) \subseteq C_{r}\left(\left(\Sigma \oplus X_{n}\right)_{p}, Y\right)$ if and only if, for all $n$, $A\left(X_{n}, Y\right) \subseteq C_{r}\left(X_{n}, Y\right)$. Moreover $A\left(l_{p} \hat{\otimes}_{n} X\right) \subseteq C_{r}\left(l_{p} \hat{\otimes}_{n} X\right)$ if and only if $A(X, Y) \subseteq C_{r}(X, Y)$.

Remark. Recalling that $C_{1}=U$ and that $C_{\infty}=D P$, one sees that these results include and generalize the following results of Bombal [1]: Theorem 1.5 , part a) for the unconditionally converging operators $(p=1$, 
$r=1$ in Lemma 4) and Dunford-Pettis operators ( $p=1, r=\infty$ in Lemma 4; Corollary 1.6. part a) for the unconditionally converging operators $(p=1$, $r=1$ in Proposition 5) and Dunford-Pettis operators ( $p=1, r=\infty$ in Proposition 5) and Proposition 2.6. a) $(p=1, r=\infty, A=L)$, and c) $(p=1$, $r=1, A=L)$; this last case also appears in [4].

Theorem 6. Let $1 \leq p<+\infty$. Assume that $r<p^{*}$ (or $p=1$ and $r=\infty)$ : these are the cases when $l_{p}$ has $D P P_{r}$. Assume that $X$ also has $D P P_{r}$. Then $l_{p} \hat{\otimes}_{r} X$ and $l_{p} \hat{\otimes}_{\Delta_{p}} X$ also have DPP.

Proof. Let $E$ denote any of those spaces, and let $T: E \rightarrow Y$ be a weakly compact operator. Since $X$ has $D P P_{r}$ the operators $\left(T_{k}\right)$ in the representing sequence of $T$, which necessarily are weakly compact, are $p$ converging. By Lemma $4, T$ must also be $p$-converging.

\section{References}

[1] F. BombaL: Operators on vector sequence spaces, London Math. Soc. Lecture Notes 140 (1989) 94-106.

[2] J. M. F. CASTILLO: p-converging and weakly-p-compact operators in $L_{p}$ spaces, Extracta Math. volume dedicated to the II Congress in Functional Analysis held in Jarandilla de la Vera, Cáceres, June 1990. (1992) 46-54.

[3] J. M. F. CASTILlo and F. SÁnCHEZ: Dunford-Pettis-like properties of continuous function vector spaces, Rev. Mat. Univ. Complutense de Madrid V. 6 , núm. 1 (1993) 43-59.

[4] J. M. F CASTILLo and J. A. López Molina: Operators defined on projective and natural tensor products, Michigan Math. J. 40 (1993), 4l 1-414.

[5] J. S. COHEN: Absolutely P-summing, P-nuclear operators and their conjugates, Math. Ann. 201 (1973) 177-200.

[6] S. Goes and R. Welland: Compactness criteria for Köthe spaces, Math. Ann. 188 (1970) 251-269.

[7] H. JARChow: Locally Convex Spaces, B.G. Teubner Stuttgart. 1981.

[8] I. LeONARD: Banach sequence spaces, J.Math. Anal. and Appl. 54 (1976) $245-256$.

Departamento de Matemáticas

Universidad de Extremadura

Avda. de Elvas, s/n.

06071 Badajoz. España.

Departamento de Matemáticas

E.T.S. Ingenieros Agrónomos

Camino de Vera, $s / n$.

Recibido: 10 de noviembre de 1992

46022 Valencia. España.

Revisado: 15 de marzo de 1993. 